\title{
A Study on Dynamic Quality Manage- ment Model for E-Commerce
}

\author{
Jinling Chang ${ }^{1}$ Chuncan $\mathbf{L i}^{2}$ \\ ${ }^{1}$ ZhengZhou Institute of Aeronautical Industry Management, Zhengzhou, China, \\ Email: chang_jinling@zzia.edu.cn \\ ${ }^{2}$ ZhengZhou Institute of Aeronautical Industry Management, Zhengzhou, China \\ Email:lcc@zzia.edu.cn
}

\begin{abstract}
This paper constructed a dynamic and objective model of E-commerce website quality management. The key quality factors, quality characters and sub-characters from the user's perspective were discussed firstly, they formed the inherent causality relationship. Applying the Bayesian Network, the E-commerce website quality management model was shaped. The presented model can be utilized in two ways. The forward reasoning applied on the Bayesian Network could evaluate the E-commerce website quality. The backward reasoning could be utilized on quality diagnosis, quality improvement. Several examples were illustrated in this paper, and the advantages and disadvantages were discussed in the end.
\end{abstract}

Keywords: Quality Management, ECommerce, Bayesin Network, Dynamic

\section{Introduction}

E-Commerce is a booming operation pattern of enterprises. The com.cn domain named in China has already reached 173649 by December 31st, 2004 [1]. Ecommerce is stepping into development peak era. The quality of E-commerce systems has become an issue concerned by both E-commerce operators and customers. Enterprises make every effort to improve the quality of products and services and meet the customers' requirements. The competition superiority and commercial benefits can be gained by constantly enhancing the quality of E-commerce systems.

The purpose of this paper is to establish an E-commerce system management model based on Bayesian network to assist E-commerce operators, developers, customers and researchers in evaluating and managing the E-commerce systems' quality.

\section{Theoretical Basis}

\subsection{The quality management of $\mathbf{E}-$ commerce website}

In the E-commerce environment, customers' requirements on the quality of products and services have increased. The customer-centered service quality administration has become the emphasis of quality administration in the whole enterprise. The final goal of E-commerce is not only to sell products (tangible or not) to end users but convey the value and make the customers satisfied.

The E-commerce website quality has direct influence on the systems' success [2,3]. Most current research work on Ecommerce websites quality is groping ones that validate the conclusion by the data gathered from the end user questionnaires. For example, Loiacono present WebQual $^{\mathrm{TM}}$ model and believed the web- 
site quality can be represented in twelve dimensions [4]. Aladwani \& Palvia measured the customer-recognized website quality and put forward the three dimensions of website quality, content quality, website content and website representation [5]. Barnes \& Vidgen had established WebQual website quality test tools to evaluate the quality of information-intense webs [6]. Ranganathan \& Ganapathy argued that the key attributes of B2C E-commerce websites included information content, design, secrecy and other dimensions [7]. Lin\& Lu test the enewspaper websites in the usability and reliability of the students' perception [8]. These works separately put forward some quality evaluating dimensions and validate their effectiveness and reliability through experiments. However, a common problem is that a dynamic quality management model of E-commerce websites has not been established.

The main contribution of this paper is to propose a dynamic and objective model of E-commerce website quality management based on Bayesian networks. This model can evaluate the website quality and moreover constantly improve the website quality.

The final goal of E-commerce systems is to satisfy customers. The E-commerce websites' quality includes service quality; interface quality, technical system quality and so on. Therefore, in this paper, the SERVQUAL quality framework [9] and quality dimensions in related works $[4,5,6,7,8]$ are integrated to establish the website quality evaluating indexes systems. Based on the quality perceived by end-users, five different quality factors of E-commerce website quality are formed. Every factor can be divided into different quality attributes and even detailed quality sub-attributes that can be measured directly.
A Bayesian network is a directed acyclic graph with probability explanation[10]. The nodes in the graph represent random variables while directed edges represent the relationship of these nodes. Conditional Probability Table (CPT) quantitatively describes the dependency between parent and child nodes $[11,12]$.

The modeling process based on Bayesian Networks can be divided into three phases as follows.

Phase1: Determine and establish the variables and explanations related to models.

A) Set the model's goal, that is set the explanation of related issue.

B) Set the related possible observation values and determines the sub sets used to establish the mode.

C) Compose these observation values into variables that can cover all states.

Phase2: Establish a directed cyclic graph that has the consistent conditional independency. have

According to probability formulas, we

$$
\begin{aligned}
p(x) & =\prod_{i=1}^{n} p\left(x_{i} / x_{1}, x_{2}, \ldots, x_{i-1}\right) \\
& =p\left(x_{1}\right) p\left(x_{2} / x_{1}\right) p\left(x_{3} / x_{1}, x_{2}\right) \ldots p\left(x_{n} / x_{1}, x_{2}, \ldots x_{n-1}\right)
\end{aligned}
$$

For each variable $x_{i}$, if there is a set $\prod_{i}=\left\{X_{1}, X_{2}, \ldots, X_{i-1}\right\}$

$$
\text { that } x_{i} \text { and } \prod_{i}=\left\{X_{1}, X_{2}, \ldots, X_{i-1}\right\} \text { are }
$$

conditional independent, that is for any $X$,

$p\left(x_{i} / x_{1}, x_{2}, \ldots, x_{i-1}\right)=p\left(x_{i} / \pi_{i}\right) i=1,2, \cdots, n$

From formula 1 and 2, we get

$$
p(x)=\prod_{i=1}^{n} p\left(x_{i} / \pi_{i}\right)
$$

\subsection{Bayesian Net}


The variable set $\left\{\Pi_{1}, \Pi_{2}, \cdots, \Pi_{n}\right\}$ is corresponding to the parent nodes $\left\{p_{a 1}, p_{a 2}, \cdots, p_{a n}\right\}$.

Therefore, when the Bayesian networks topology structure building up, we (1) sort the variables $X_{1}, X_{2}, \cdots, X_{n}$ to certain order; (2) find the variable set $\Pi_{i}(i=1,2, \cdots, n)$ that satisfies formula 2.In the application of Bayesian networks, it is common that real world problem determine the causality relationship, while causality relationship usually has the consistent conditional independency. So an arrowed arch can be drawn from cause variable to effect variable in order to apparently represent the causal relationship among variables.

Phase 3: Assign the probability distributions $p\left(x_{i} / p_{a i}\right)$. In the discrete situation, every variable $x_{i}$ that has parent nodes need to be assigned its probability distribution.

In solving many real world problems, we need to reason from approximate and incomplete knowledge and information. While A Bayesian network is a probability reasoning technology, based on probability theory, it deals with uncertainties generated by describing the conditional dependency between knowledge compositions. It provides a viewable approach presenting knowledge in graphs.

\section{E-Commerce Website Quality Model}

The Bayesian network model contains two presumptions, the non-circle presumption (we suppose that the network topology does not have circles) and the static state presumption (the lag time between the cause nodes and the effect nodes is not considered)[10]. In building E-commerce website quality model, the circle structures can be avoided by rea- sonable selection of nodes in Bayesian. In this paper, the nodes selected when establishing the model are time independent, so the problem of time lagging does not exist. The presumption of static states is satisfied.

\subsection{Set the $B N$ nodes}

The overall Quality of the E-commerce website systems is set as the modeling goal and thus becomes the central nodes of the network.

According to the literature study, the overall quality of the E-commerce website based on the quality perceived by the end-users is represented by five quality factors: tangibility factor, reliability factor, response factor, security factor and guarantee factor and empathy factor. Every quality factor can also have several quality attributes and each quality attribute can have sub-attributes. This quality sub-attribute $\rightarrow$ quality attribute $\rightarrow$ quality factor $\rightarrow$ overall quality model naturally represents the causal relationship among these variables and satisfies the consistent conditional independency. Therefore, the established Bayesian Network topology structure for Ecommerce website quality administration is shown in figure 1.

\subsection{Set the apriority probability and CPT}

After establishing the Bayesian Network topology structure, we need to determine the value of every node in the network structure and the CPT of the variables represented by non-root nodes. The dependency relationship among variables needs to be quantified.

In ISO9000: 2000 the quality is defined as follows. 'Quality is the degree to which a group inherent attributes meet the requirements'. "Degree" can be measured by "good and poor". So we define three possible states for every quality 
factor, quality attribute: Good (G), Aver- evidence of every child-node. age (A), and Poor (P). This is also the

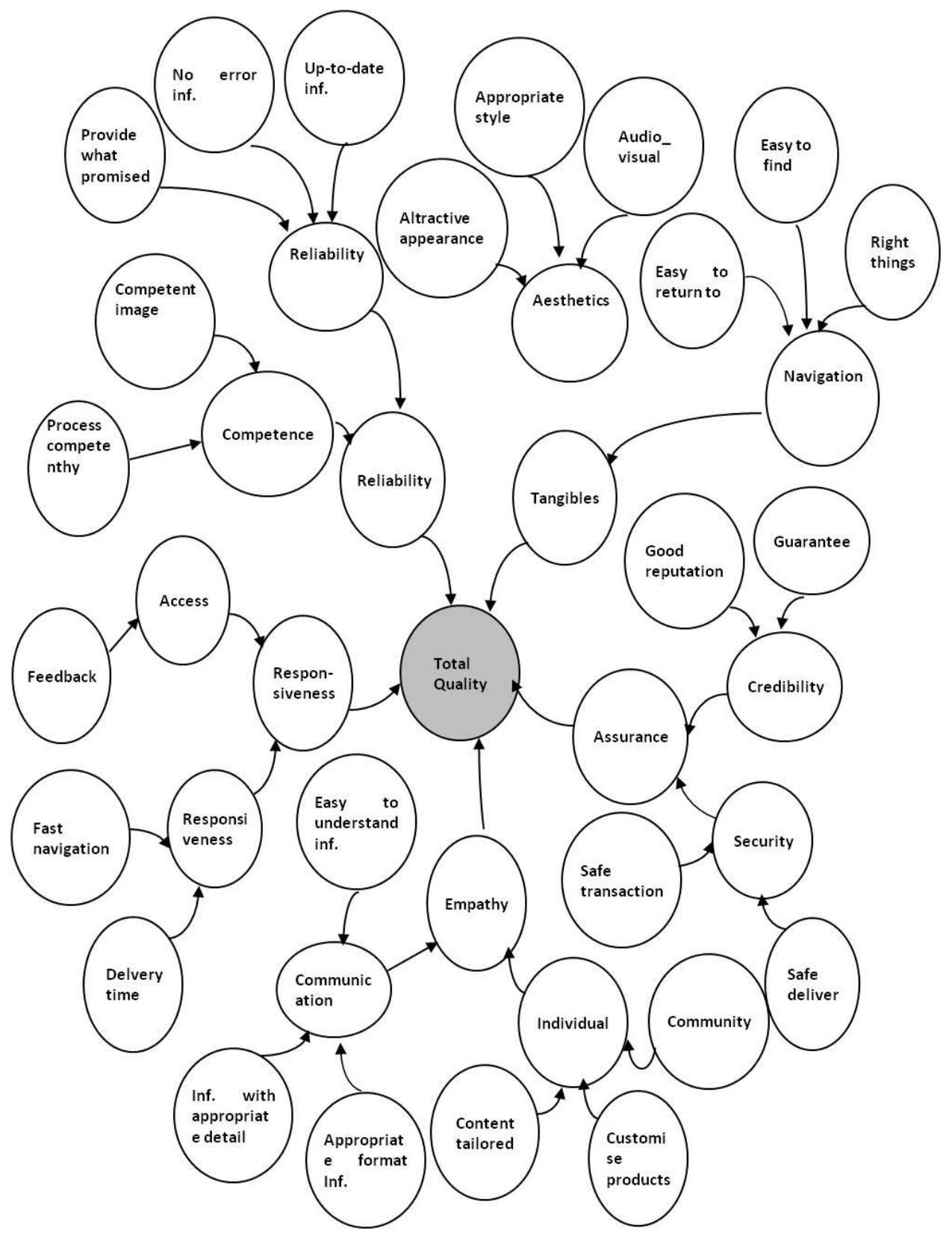

Fig.1: E-commerce website quality management model structure.

The priority probability comes from the users. The users only need simply an- swer "yes" or "no" whether this Ecommerce website have such quality sub- 
attributes. This can too much extent reduce the subjectivity of the evaluating.

For each child-node that has parentnodes, the discrete conditional probability distribution represented by CPT denotes the quantitative dependency between this child-node and its parent nodes. In this paper, the values of CPT are assigned by the specialists.

\subsection{The dynamic characteristic of the model}

The quality administration model established above have the dynamic characteristics. This is because of the nodes' CPT. The initialized CPT value is set by specialists according to their knowledge and experiences. This model can continuously improve the submitted results through case learning. Based on systematical exact data collection, CPT enables the model to provide exact evaluation results.

\section{Quality Management of E- commerce Website}

\subsection{Quality evaluation}

The E-commerce website quality model can conduct forward reasoning and also backward reasoning.. Forward reasoning is a top-down computing which get the probability of results based on some known evidence using Bayesian Networks. For quality administration, it is the process of evaluating the quality of Ecommerce website systems [13].

For example, quality attribute 'Security' has two parent nodes, also called two quality sub-attributes, 'Safe transaction' and 'Safe deliver'. If 42 percent users in the questionnaire believe that the evaluated website has the quality subattribute 'Safe transaction' and 87 percents believe that the evaluated website has the quality sub-attribute 'Safe deliver', shown in figure 2 , then based on the CPT of 'Security' node we can get that the probability that the website quality attribute 'Security' can be evaluated as 'good' is 57 percent, 'average' $21 \%$ and 'poor' $22 \%$.

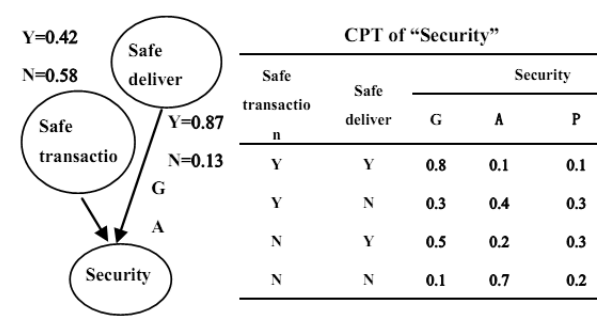

Fig. 2: Quality management.

After the quality attributes evaluation is done, the quality factor evaluation can also be conducted in the same reasoning process. Consequently, the overall quality can finally be evaluated.

The E-commerce website quality Bayesian network model can assist the website developers to evaluate the importance of quality attributes and quality factors, and also the mutual influences between parent codes and child codes. For example, if the developers want to evaluate how some extent sub-attributes, such as 'Provide what promised', 'No error inf.', and 'Up-to-date inf.', influence the quality attribute 'Reliability', then they can assign different evident values in order to get different 'Reliability' results. As it is shown in figure 3 , these different outcomes can help designers to decide which sub-attribute influences 'reliability' more.

It is notable that even in case that the evidence is not enough, the Bayesian network can still conduct reasoning by recovering datasets, and approximate computing, etc. But precision of quality evaluation is higher with more evidence in the model. 


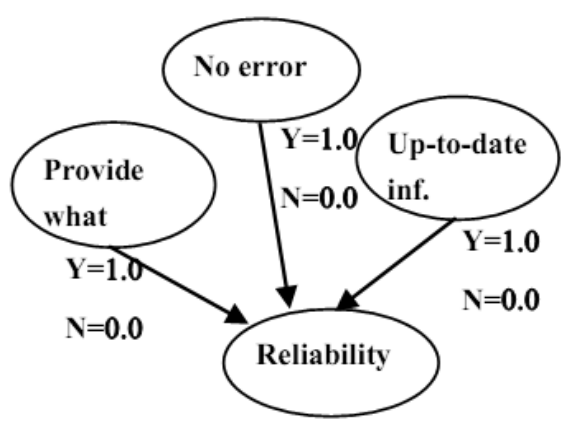

Fig. 3: Another sample.

\subsection{Quality diagnosis}

The backward reasoning of Bayesian networks is to deduce the cause from the effects. It is a kind of bottom-up reasoning and also called diagnosis reasoning. Given the results, based on the Bayesian network, the backward reasoning educes the probability of the cause.

Suppose that in the diagnosis process the evidence is $e$, then the edge probability of any node A can be calculated as below.

$$
\begin{aligned}
p(A) & =\frac{p(A, e)}{p(e)} \\
= & \frac{\sum_{U /\{A\}} p(U, e)}{p(e)}
\end{aligned}
$$

For example, given that 'navigation' of an E-commerce website is 'good' and the probability of other related sub-attributes is small, it can be calculated through the model that 'easiness to locate' has the probability more than $85 \%$ to be 'good'.

Similarly, given that the 'overall quality' of an E-commerce website is 'good' and the quality factor 'reliability' is 'good', by backward reasoning, we can get that the probability distribution of quality attribute 'provide newest attributes' is 'good' $80 \%$, 'average' $16 \%$, 'poor' 4\%. Therefore, in the backward reasoning, assigning the evidence value of child node can provide probability value of its related parent nodes. The developers of E-commerce utilize these to conduct quality diagnosis, continuous quality improvement and reasonable resource distribution.

\section{Conclusion}

This paper establishes E-commerce website quality administration model based on Bayesian network. This model is objective and dynamic. Compared with other research approaches, this model not only can evaluate the website quality but also can conduct quality diagnosis and continuous improvement.

The establishment of Bayesian network topology model in this paper is based on previous research works. The function dependency relationships between variables (CPT) are set by specialists, which is a point that needs improvement in this paper.

The Bayesian Network also has the learning functionality, dynamically build up the networks from real cases. If case data is enough, Bayesian learning can automatically build the most suitable Bayesian networks to these real case data. The CPT of every child node can be determined by case training after the specialists have build up the network topology structure. It is also one of the future research works.

\section{References}

[1] http://www.cnnic.cn/index/0E/00/11/i ndex.htm.

[2] Gholamreza Torkzadeh, Gurpreet Dhillon, Measuring Factors that Influence the Success of Internet Commerce[J], Information System Research, 13(2), pp. 187 - 204, 2002

[3] Vicki McKinney , Kanghyun Yoon, The Measurement of Web-Customer 
Satisfaction: An Expectation and Disconfirmation Approach[J], Information System Research, 13(3), pp. 296315, 2002

[4] E.T. Loiacono, WebQualTM: a website quality instrument, Doctoral dissertation, University of Georgia[D], Athens, 2000

[5] A.M. Aladwani, P.C. Palvia, Developing and validating an instrument for measuring user-perceived web quality[J], Information \& Management, 39, pp. 467-476, 2002

[6] S.J. Barnes, R.T. Vidgen, An evaluation of cyber-bookshops: the WebQualTM method [J], International Journal of Electronic Commerce, 6 (1), pp. 11-30, 2001

[7] C.Ranganathan, S.Ganapathy, Key dimensions of business to-consumer websites[J], Information \& Management, pp. 457-465, 2002

[8] J.C. Lin, H. Lu, Towards an understanding of the behavioral intention to use a website[J], International Journal of Information Management, pp.197208, 2002

[9] Parasuraman A, Zeithaml V A, Berry L L. SERVQUAL: A multiple -item scale for measuring consumer perceptions of service quality[J]. Journal of Retailing, 64(1), pp. 12-40, 1998

[10] Bayes. T.R. An essay towards solving a problem in the doctrine of chances[J], Philosophical Transactions, pp. 370-418, 1763

[11] Pearl J. Fusion, Propagation and structuring in belief networks[J], Artificial Intelligence, 29(3), pp.241288,1986

[12]Pearl J., Probabilistic Reasoning in Intelligent Systems: Networks of Plausible Inference[M], Morgan Kaufmann, San Francisco, (revised in 1997).

[13] A.Stefani, M. Xenos, D.Stavrinoudis, Modeling E-Commerce Systems' Quality with Belief Networks[A].
Proceeding of VECIMS 2003International Symposium on Virtual Environments, Human-Computer Interfaces, and Measurement Systems [C] ,2002 